\title{
ENSAIO COM SULPIRIDE EM ESQUIZOFRENICOS HOSPITALIZADOS
}

\author{
Clóvis MaRTins * \\ DARLAN F. NeVES ** \\ Gabriel A. S. Gama ** \\ Hilton R. Gavioli ** \\ Imeuda M. A. Carvalho **
}

\author{
Marco antonio N. Amaral *: \\ NELSON C. VIEIRA ** \\ RONALDO B. Oliveira ** \\ WILMA G. LEAL ** \\ Luís M. Assis ***
}

O sulpiride tem sido usado experimentalmente em psiquiatria 2, 5, 6, 7, 10, em pediatria ${ }^{3,9}$, em afecções do trato gastrintestinal ${ }^{8}$, em sindromes vertiginosas $^{4}$ e em neurocirurgia ${ }^{1}$. O sulpiride é a $-\mathrm{N}-1$ (1-etil-2-metil) pirrolidinil | 2 metoxi-5-sulfamoil benzamida. As propriedades dessa droga a enqudram entre os neurolépticos e os timoanalépticos: é antiemética, neuroléptica, antidepressiva e não tem ação sedativa. No tratamento das psicoses apresenta propriedades antialucinatória, anticonfusional, com ação rápida e duradoura, exibindo leve ação extrapiramidal.

\section{CASUfSTICA, MẼTODOS E RESULTADOS}

Propusemo-nos a estudar o efeito do sulpiride em pacientes esquizofrênicos internados. $O$ sulpiride * foi experimentado em 24 pacientes, com idades entre 17 e 48 anos, sendo 17 de sexo masculino e 7 de sexo feminino. Todos tinham diagnóstico de esquizofrenia. O tempo de doença variou de 30 dias a 18 anos, sendo de notar que na maioria dos casos esse tempo foi superior a três anos.

o desenvolvimento da pesquisa obedeceu ao seguinte esquema: a) resumo das observaçōes, sendo destacado o último tratamento; $b$ ) o tratamento prévio, se existente, foi suspenso pelo menos 10 dias antes do início da investigacão; $c$ ) foi realizado exame psíquico completo antes do início do ensaio, sendo quantificados os dados em tabela; d) o exame psíquico foi repetido e quantificado semanalmente, até o término da experimentação; e) a duração do tratamento foi de 6 semanas em média; f) foi feito controle diário de pulso, pressão arterial e temperatura.

Foram utilizadas ampolas de $100 \mathrm{mg}$ e comprimidos de $200 \mathrm{mg}$ de substância ativa, segundo o seguinte esquema: $100 \mathrm{mg}$ cada 6 horas no primeiro, segundo e terceiro dias; $200 \mathrm{mg}$ cada 8 horas no quarto, quinto, sexto e sétimo dias. A partir da segunda semana a dose foi mantida ou ajustada segundo o caso, tendo a dose máxima diária atingido $1600 \mathrm{mg}$.

Levando em conta as entrevistas médicas e as anotacões de enfermagem, foram registrados apenas os efeitos colaterais referidos espontaneamente pelos pacientes.

Trabalho realizado no Sanatório Anhembi: * Diretor Clínico; ** Médicos psiquiatras; *** Médico neurologista.

* Medicamento cedido pelos Laboratórios Lepetit S.A. - Nome comercial: Equilid. 
A identificação dos casos estudados e os resultados obtidos podem ser observados nos quadros 1 e 2 . Os pacientes 1 e 4 evadiram-se no décimo-quinto e no décimo-quarto dias de tratamento, respectivamente. O paciente 10 foi transferido no décimo-quarto dia para tratamento especializado de tuberculose pulmonar. Nos casos 18 e 20 o tratamento foi suspenso em virtude de pioras, especialmente em decorrência do aumento da excitação. Não foram observados efeitos colaterais importantes durante o ensaio terapêtico.

\begin{tabular}{|c|c|c|c|c|c|c|}
\hline Caso & Nome & Registro & $\begin{array}{c}\text { Diagnóstico } \\
\text { (CID-8) }\end{array}$ & $\begin{array}{l}\text { Duração } \\
\text { (dias) }\end{array}$ & $\begin{array}{l}\text { Dose diária } \\
\text { (mg) }\end{array}$ & $\begin{array}{c}\text { Evolução } \\
0-1-2\end{array}$ \\
\hline 1 & I. F. S. & 22.826 & 295.3 & 15 & 600 & 0 \\
\hline 2 & F. J. S. & 21.954 & 295.3 & 37 & 600 & 2 \\
\hline 3 & J. G. & 6.857 & 295.3 & 50 & 1200 & 0 \\
\hline 4 & R. T. S. & 21.974 & 295.3 & 10 & 600 & 1 \\
\hline 5 & A. G. L. & 22.129 & 295.3 & 45 & 1600 & 2 \\
\hline 6 & R. G. & 22.258 & 295.3 & 40 & 1600 & 0 \\
\hline 7 & S. V. S. & 21.587 & 295.3 & 45 & 1200 & 1 \\
\hline 8 & S. L . Z . & 21.817 & 295.3 & 45 & 1200 & 2 \\
\hline 9 & A. C. C. & 21.855 & 295.3 & 45 & 1200 & 1 \\
\hline 10 & J. D. S. A. & 21.990 & 295.3 & 14 & 1200 & 1 \\
\hline 11 & J. R. S. & 22.799 & 205.3 & 52 & 1400 & 0 \\
\hline 12 & E. A. & 22.587 & 295.3 & 60 & 600 & 2 \\
\hline 13 & C. P. M. & 23.106 & 295.3 & 45 & 600 & 2 \\
\hline 14 & S. Q. A. T. & 23.624 & 295.3 & 45 & 1200 & 2 \\
\hline 15 & W. L. S. & 21.795 & 295.3 & 45 & 1200 & 2 \\
\hline 16 & A. C. N. & 23.138 & 295.3 & 43 & 1200 & 2 \\
\hline 17 & A. J. & 23.444 & 295.3 & 36 & 800 & 0 \\
\hline 18 & $\ddot{\mathrm{u}} \cdot \mathbf{A} \cdot \mathbf{M}$. & 23.534 & 295.3 & 26 & 800 & 0 \\
\hline 19 & P. A. A. & 23.720 & 295.3 & 45 & 1600 & 2 \\
\hline 20 & E. B. F. & 23.748 & 295.3 & 21 & 1200 & 0 \\
\hline 21 & V. L. S. & 23.587 & 295.3 & 45 & 800 & 1 \\
\hline 22 & P. V. H. & 23.821 & 295.3 & 70 & 800 & 2 \\
\hline 23 & R. C. & 23.737 & 295.3 & 45 & 800 & 2 \\
\hline 24 & E. A. & 23.721 & 295.3 & 45 & 800 & 0 \\
\hline
\end{tabular}

Quadro 1 - Identificacão dos pacientes, diagnóstico, duracão do tratamento, dose diária máxima e evolução dos casos estudados: $0=$ inalterado; 1 = melhora; 2 = melhora notável ou remissão dos sintomas. 
Caso

Dellrios
Aluci- Desagre- Autis- Contato Apragnações gação

\section{mo deficiente matismo}

Exci- Catatonia tação

\begin{tabular}{|c|c|c|c|c|c|c|c|c|}
\hline $1^{A}$ & +++ & +++ & +++ & +++ & +++ & +++ & - & - \\
\hline D & +++ & ++ & ++ & ++ & ++ & ++ & - & - \\
\hline $2^{A}$ & +++ & ++ & +++ & + & ++ & ++ & - & - \\
\hline D & - & - & - & - & + & + & - & - \\
\hline $3^{A}$ & - & - & ++ & ++ & ++ & +++ & - & - \\
\hline D & - & - & ++ & ++ & ++ & +++ & - & - \\
\hline $4^{\mathrm{A}}$ & +++ & +++ & ++ & +++ & + & +++ & + & - \\
\hline D & +++ & ++ & ++ & ++ & + & ++ & + & - \\
\hline $5^{A}$ & - & - & +++ & ++ & + & +++ & ++ & - \\
\hline D & - & - & + & + & + & + & - & - \\
\hline $6^{A}$ & ++ & ++ & 一 & + & +++ & +++ & - & - \\
\hline D & ++ & ++ & - & + & +++ & ++ & - & - \\
\hline $7^{A}$ & 一 & - & - & ++ & +++ & +++ & - & ++ \\
\hline D & - & - & - & - & + & +++ & - & - \\
\hline $8^{A}$ & - & - & - & - & ++ & ++ & - & - \\
\hline D & - & - & - & - & - & + & - & - \\
\hline $9^{\mathbf{A}}$ & - & - & - & +++ & +++ & +++ & - & +++ \\
\hline D & - & - & - & +++ & + & +++ & - & - \\
\hline $10^{\mathrm{A}}$ & +++ & +++ & ++ & +++ & +++ & +++ & +++ & - \\
\hline D & ++ & + & ++ & ++ & + & +++ & ++ & - \\
\hline $11^{A}$ & +++ & - & - & - & - & + & - & - \\
\hline D & +++ & - & - & - & - & + & - & - \\
\hline $12^{\mathrm{A}}$ & +++ & - & - & - & - & - & - & - \\
\hline D & - & - & - & - & - & -- & - & - \\
\hline $13^{A}$ & ++ & ++ & - & - & ++ & ++ & - & - \\
\hline D & 一 & $\rightarrow$ & - & - & - & - & - & - \\
\hline $14^{\mathrm{A}}$ & +++ & +++ & +++ & +++ & +++ & +++ & ++ & - \\
\hline D & - & - & - & - & - & - & - & - \\
\hline
\end{tabular}




\begin{tabular}{|c|c|c|c|c|c|c|c|c|}
\hline Caso & $\begin{array}{l}\text { Delí- } \\
\text { rios }\end{array}$ & $\begin{array}{l}\text { Aluci- } \\
\text { nações }\end{array}$ & $\begin{array}{c}\text { Desagre- } \\
\text { gação }\end{array}$ & $\begin{array}{c}\text { Autis- } \\
\text { mo }\end{array}$ & $\begin{array}{l}\text { Contato } \\
\text { deficiente }\end{array}$ & $\begin{array}{l}\text { Aprag- } \\
\text { matismo }\end{array}$ & $\begin{array}{l}\text { Exci- } \\
\text { tação }\end{array}$ & Catatonia \\
\hline A & +++ & +++ & +++ & +++ & ++ & ++ & +++ & 一 \\
\hline${ }^{10} \mathrm{D}$ & - & - & 一 & + & - & + & - & - \\
\hline A & +++ & - & - & + & ++ & 一 & - & 一 \\
\hline \multicolumn{9}{|l|}{16} \\
\hline D & 一 & - & 一 & 一 & - & 一 & 一 & - \\
\hline A & 一 & 一 & - & + & ++ & ++ & ++ & - \\
\hline \multicolumn{9}{|l|}{17} \\
\hline D & 一 & 一 & - & + & ++ & ++ & + & 一 \\
\hline A & ++ & - & ++ & - & + & ++ & + & - \\
\hline \multicolumn{9}{|l|}{18} \\
\hline D & ++ & 一 & ++ & 一 & + & ++ & +++ & - \\
\hline A & +++ & ++ & +++ & +++ & +++ & +++ & +++ & - \\
\hline \multicolumn{9}{|l|}{19} \\
\hline D & 一 & 一 & $\rightarrow$ & + & + & + & 一 & 一 \\
\hline A & ++ & ++ & - & +++ & ++ & ++ & ++ & - \\
\hline \multicolumn{9}{|l|}{20} \\
\hline D & ++ & ++ & $\cdots$ & +++ & +++ & +++ & +++ & - \\
\hline${ }_{21}{ }^{\mathrm{A}}$ & - & - & ++ & + & ++ & ++ & 一 & - \\
\hline $\mathrm{D}$ & 一 & 一 & + & + & + & + & - & 一 \\
\hline A & - & 一 & + & + & ++ & ++ & 一 & 一 \\
\hline \multicolumn{9}{|l|}{22} \\
\hline D & 一 & 一 & 一 & - & 一 & - & - & - \\
\hline A & + & + & ++ & + & +++ & ++ & ++ & - \\
\hline \multicolumn{9}{|l|}{23} \\
\hline D & - & - & - & - & + & 一 & - & - \\
\hline A & 一 & 一 & +++ & ++ & + & + & + & - \\
\hline 24 & & & & & & & & \\
\hline D & - & 一 & +++ & ++ & + & + & - & - \\
\hline
\end{tabular}

Quadro 2 - Avaliação dos sintomas, antes e depois do tratamento. Legenda: $A=$ antes; $D=$ depois; $+++=$ intenso; $++=$ médio; $+=$ discreto; $-=$ ausente.

\section{COMENTÃRIOS}

Como tem sido referido na literatura, o sulpiride foi empregado em diversas afecções, de natureza variada. Em Psiquiatria seu uso é referido em maior escala. Atruz ${ }^{2}$ verificou ter a droga pequeno efeito sedativo, sendo realçados efeitos antimaníacos, assim como ação antidepressiva; além disso esse autor constatou ação nas desordens mentais esquizofrênicas. Naymanovich ${ }^{5}$, estudando 200 pacientes mentais esquizofrênicos e delirantes, con- 
cluiu ser o sulpiride um neuroléptico maior e timoanaléptico com ação desinibidora, embora sua atividade nas esquizofrenias fosse considerada irregular. Pinto ${ }^{6}$ fez ensaio duplo-cego em dois grupos de pacientes esquizofrênicos, comprovando a ação benéfica da droga em cerca de $80 \%$ dos casos; sua ação mais evidente foi delírio e alucinolítica, sendo notada melhora também no pragmatismo. Finalmente Toru \& col. ${ }^{10}$ estudaram o sulpiride comparativamente à clorpromazina em esquizofrênicos crônicos; foram analisados 38 pares de pacientes do mesmo sexo e de faixa etária semelhante. Nos resul. tados globais o sulpiride se mostrou mais efetivo que a clorpromazina em 23 pares, enquanto que a última droga foi mais efetiva em 13. Os efeitos colaterais foram semelhantes com ambas as drogas.

No sentido de reavaliar a ação dessa droga nas esquizofrenias propusemo-nos a estudar seu efeito em pacientes esquizofrênicos internados. Deve-se notar que nos casos submetidos à experimentação predominam os pacientes com longa evolução da moléstia (média de 7 anos).

Pela análise do quadro 1 verifica-se, no que diz respeito à evolução global dos casos estudados, que 8 pacientes não se beneficiaram com o tratamento, 5 apresentaram alguma melhora e 11 beneficiaram-se de maneira notável. Pela análise do quadro 2, em que se tentou fazer controle evolutivo segundo as manifestações que os pacientes exibiam, pode-se ter uma idéia da ação do medicamento. Efeito deliriolítico foi notado em 9 casos. No que se refere aos componentes senso-perceptivos, ação alucinolítica foi observada em 9 casos. Houve também alguma ação sobre a desagregação (melhora em 8 casos) e sobre o autismo (melhora em 13 casos). O contato se estabeleceu melhor no final do tratamento em 14 pacientes, sendo também notadas melhoras no apragmatismo em 13 casos.

Julgamos importante a avaliação do componente excitação dos casos estudados. Se, por um lado, melhoras puderam ser notadas em 8 casos, em dois pacientes (casos 18 e 20) a experimentação foi suspensa em virtude de pioras, especialmente em decorrência do aumento da excitação. Finalmente deve-se mencionar que em dois casos com componente catatônico, a evolução foi boa sob esse ponto de vista.

Como se pode observar pela avaliação de nossos resultados, principalmente se levarmos em conta de prevalecerem na pesquisa pacientes com longa evolução da moléstia, o sulpiride mostrou-se eficiente no tratamento das esquizofrenias, tendo agido com intensidade maior ou menor, em todas as esferas abordadas na presente investigação.

Por outro lado, não encontramos em nossos pacientes efeitos colaterais notáveis, sendo a droga praticamente destituída de poder impregnante; a tolerância por parte dos pacientes foi boa, não sendo referidas, em nenhum caso, manifestações subjetivas que pudessem estar relacionadas com a ingestão do medicamento.

Somadas estas observações aos resultados referidos na literatura, pode-se concluir que o sulpiride tem ação neuroléptica indiscutível sem efeitos colaterais indesejáveis. A droga merece ser testada em formas agudas de esquizofrenia no sentido de melhor avaliação dos seus efeitos. 


\section{RESUMO}

Um ensaio terapêutico é feito com sulpiride, substância psicotrópica dada como muito ativa e cujas propriedades permitem enquadrá-la entre os neurolépticos e os timoanalépticos. Foram tratados 24 pacientes internados, com idades que variavam entre 17 e 48 anos, de ambos os sexos (17 masculinos e 7 femininos), todos com o diagnóstico clínico de esquizofrenia e internados em hospital psiquiátrico. O tempo de doença variava de 30 dias a 18 anos. O sulpiride foi aplicado em doses diárias em torno de $1200 \mathrm{mg}$, predominantemente por via oral. O tratamento durou em média 6 semanas permanecendo a maioria dos casos em observação por alguns meses, sob tratamento de manutenção. Os resultados foram em geral favoráveis, principalmente no que tange aos fenômenos psicóticos sensoperceptivos e delirantes. Não foram assinalados efeitos colaterais ou manifestações colaterais molestas.

\section{SUMMARY}

\section{Clinical trial with sulpiride on schizophrenic in-patients}

A therapeutic trial with sulpiride was made, submiting to treatment 24 in-patients of a mental hospital, diagnosed as schizophrenics. The ages are from 17 to 48 years old, 17 males and 7 females. The time of disease was from 30 dayes to 18 years. The drug was given mainly orally on about $1200 \mathrm{mg} /$ die, in a treament of 6 weeks followed in some cases, by several months of observation. The best results were obtained in the sensoperceptive and delusional symptoms. No side effects of importance were detected.

\section{REFERENCIAS}

1. ACQUAVIVA, $R$. - Intéret $d u$ Dogmatil en pratique neurochirurgicale. J. med. maroc. 8:231, 1972 .

2. ATRUZ, M. D. - Place du sulpiride en psychopharmacologie et en pratiquepsychiatrique. J. med. chir. Prat. 143:921, 1972.

3. DIAZ, M. A. \& MACCARONE, H. E. - Asma infantil: tratamiento con sulpirida. Dia médico (Buenos Aires) 44:460, 1972.

4. KAMINSZCZIK, I.; GOLDBERG, R. \& ALENIK, L. - Investigación clínica con sulpirida. Prensa Med. Argent. 59:1170, 1972.

5. NAYMANOVICH, R. - Evaluación clinica de la sulpirida 200 en ciertas psicosis. Dia Médico (Buenos Aires) 44:652, 1972.

6. PINTO, C. A. C. - O uso do sulpiride em esquizofrenia: ensaio duplo cego. Bol. Psiquiat. (São Paulo) 5:103, 1972.

7. REBoIRAS, J. C. - El uso de sulpirida em paidopsiquiatria. Dia Médico (Buenos Aires) 44:557, 1972.

8. ROMERO, J. T. - Tratamiento del enfermo funcional digestivo: aspectos psicosomáticos del deprimido con somatización digestiva; clínica, diagnóstico y tratamiento. New England. J. Med. 6:26, 1972.

9. SPADA, C.; BEVACQUA, E.; ESTEYBAR, C. \& PERELLO, L. - Experiencia argentina con sulpirida en pediatria. Arch. Arg. Ped. 70:193, 1972.

10. TORU, M.; SHIMAZONO, Y.; NIYASAKA, M.; KOKUBO, T.; MORI, Y. \& NASU, T. - A double-blind comparison of sulpiride with chlorpromazine in chronic schizophrenia. J. clin. Pharm. 12:221, 1972.

Sanatório Anhembi - Rua Desembargador Vale, 938 - 05010 São Paulo, SP - Brasil. 